\title{
Effect of estrogens on boar sperm capacitation in vitro
}

\author{
Lukas Ded', Pavla Dostalova1, Andriy Dorosh¹, Katerina Dvorakova-Hortova² and Jana Peknicova*1
}

\begin{abstract}
Background: Mammalian sperm must undergo a series of controlled molecular processes in the female reproductive tract called capacitation before they are capable of penetrating and fertilizing the egg. Capacitation, as a complex biological process, is influenced by many molecular factors, among which steroidal hormone estrogens play their role. Estrogens, present in a high concentration in the female reproductive tract are generally considered as primarily female hormones. However, there is increasing evidence of their important impact on male reproductive parameters. The purpose of this study is to investigate the effect of three natural estrogens such as estrone (E1), 17beta-estradiol (E2) and estriol (E3) as well as the synthetical one, 17alpha-ethynylestradiol (EE2) on boar sperm capacitation in vitro.

Methods: Boar sperm were capacitated in vitro in presence of estrogens. Capacitation progress in control and experimental samples was analyzed by flow cytometry with the anti-acrosin monoclonal antibody (ACR.2) at selected times of incubation. Sperm samples were analyzed at 120 min of capacitation by CTC (chlortetracycline) assay, immunocytochemistry and flow cytometry with anti-acrosin ACR.2 antibody. Furthermore, sperm samples and capacitating media were analyzed by immunocytochemistry, ELISA with the ACR.2 antibody, and the acrosin activity assay after induced acrosomal reaction (AR).
\end{abstract}

Results: Estrogens stimulate sperm capacitation of boar sperm collected from different individuals. The stimulatory effect depends on capacitation time and is highly influenced by differences in the response to estrogens such as E2 by individual animals. Individual estrogens have relatively same effect on capacitation progress. In the boar samples with high estrogen responsiveness, estrogens stimulate the capacitation progress in a concentration-dependent manner. Furthermore, estrogens significantly increase the number of acrosome-reacted sperm after zona pellucida- induced acrosomal reaction.

Conclusions: We demonstrate here the stimulatory effect of four different estrogens on boar sperm capacitation in vitro. According to our results, there is significant difference in the response to tested estrogens at different capacitation time and among individual animals. In animals with a high response to estrogens, there is a concentration-dependent stimulation of capacitation and individual estrogens have relatively the same effect. Effects of individual estrogens, differences in the response to them by individual animals, their time and concentrationdependent outcomes further contribute to our knowledge about steroidal action in sperm.

\section{Background}

Capacitation involves the physiological changes that spermatozoa must undergo in the female reproductive tract or in vitro to obtain the ability to penetrate and fertilize the egg [1-3]. Capacitation is a complex molecular process that results in changes of calcium concentration,

* Correspondence: jpeknic@biomed.cas.cz

${ }^{1}$ Laboratory of Diagnostics for Reproductive Medicine, Institute of Biotechnology, Academy of Sciences of the Czech Republic, v. v. i., Prague, Czech Republic

Full list of author information is available at the end of the article protein phosphorylation, acrosomal matrix and membrane rearrangement. As a complex biological process, capacitation can be influenced by many molecular factors in the uterine and oviductal fluid [4] and the effect of uterine and oviductal fluids depends on the specific stages of the estrous cycle [5]. Although capacitation naturally occurs in the female reproductive tract, it can be also performed in vitro using specific media and physical conditions [6,7].

Estrogens are a group of steroid compounds, named for their importance in the estrous cycle. Although estrogens 
have been considered mainly female reproductive hormones, they also play an important role in regulating male reproductive functions. The main breakthrough in this field was brought forth by estrogen receptor knockout mice. Phenotypically, these mice have significant alteration in testes histology, spermiogenesis and they suffer from infertility [8].

In somatic cells, estrogens act through three known estrogen receptors - ERa, ERb and GPR30. ERa and ERb are called - classical estrogen receptor. They bind specific loci in DNA (estrogen response elements) and act as transcriptional factors. Recently, there has been evidence of a nongenomic effect of these receptors [9] and this effect may be important for estrogen regulation of the sperm function since sperm are supposed to be transcriptionally silent. Classical estrogen receptors were found in human spermatozoa and there is evidence for their direct interaction with phosphatidylinositol-3-OHKinase/Akt pathway [10]. This observation is important, because some receptors in sperm membrane are supposed to have only a passive role [11]. Classical estrogen receptors were recently found together with the aromatase and androgen receptor in pig spermatozoa [12]. Beside classical receptors, estrogens can act through the membrane estrogen receptor GPR30. GPR30 signalization is accompanied by calcium mobilization, therefore, a signalization through this receptor seems to be a good candidate for estrogen pathway in sperm. However, to this date there is no evidence for the presence of this receptor in the sperm. Finally, there is some evidence for the presence of putative estrogen receptors in the sperm, which is different from the classical ones. The antibodies against these putative receptors block the stimulatory effect of estrogens but their functions remain to be elucidated [13].

Although several studies report effects of estrogen in mature spermatozoa, there are some contradictory results in this field. There are a few papers from $1970 \mathrm{~s}$ $1980 \mathrm{~s}$ concerning the effect of estrogens and progesterone on capacitation of hamster and rabbit sperm in vivo and in vitro. Gwatkin and Williams reported an inhibitory effect of the follicular fluid enriched by progesterone and estrogens on capacitation of rabbit spermatozoa in vitro [14]. Briggs obtained similar results with hamster sperm [15]. Contrary to this, Bathla et al. reported a significantly higher number of spermatozoa incubated in isolated uterus enriched by exogenous estrogens [16]. Further, Hamner and Wilson concluded that antiestrogens have no effect on the capacitation progress of rabbit sperm [17]. Recently, it was reported that there is a stimulatory effect of estrogens and different xenoestrogens on capacitation, acrosome reaction and fertilizing ability of mouse spermatozoa [18]. Furthermore, pre-incubation with estrogens does not alter the ability of human sperm to fuse with the oocyte [19].
In this study, we investigated the effect of three natural estrogens such as estrone (E1), 17 $\beta$-estradiol (E2), estriol (E3), and one syntetical estrogen (17 $\alpha$-ethynylestradiol, EE2) on capacitation and AR of boar sperm in vitro.

\section{Methods \\ Chemicals}

All chemicals were purchased from Sigma (Prague, Czech Republic) unless otherwise specified.

\section{Sperm capacitation in vitro and calcium ionophore/zona pellucida-induced acrosomal reaction}

Boar (Sus scrofa) ejaculates were supplied by Insemination Station, Kout na Sumave, CR. All sperm samples were examined for their motility and viability. Samples of poor quality were discarded. Suitable sperm samples were washed twice in tris-buffered saline (TBS, $200 \times \mathrm{g}$, $10 \mathrm{~min}$ ), centrifuged on Percoll gradient (80, 70, 55, 40\% Percoll, $200 \times \mathrm{g}, 60 \mathrm{~min}$ ) and washed in capacitation medium without bovine serum albumine $(11.3 \mathrm{nM} \mathrm{NaCl}$, 0,3 $\mathrm{mM} \mathrm{KCl}, 1 \mathrm{mM} \mathrm{CaCl}$, $2 \mathrm{mM}$ TRIS, $1.1 \mathrm{mM}$ glucose, $0.5 \mathrm{mM}$ pyruvate). After being washed and percolled, sperm were resuspended in capacitation medium (11.3 $\mathrm{nM} \mathrm{NaCl}, 0.3 \mathrm{mM} \mathrm{KCl}, 1 \mathrm{mM} \mathrm{CaCl}, 2 \mathrm{mM}$ TRIS, 1.1 $\mathrm{mM}$ glucose, $0.5 \mathrm{mM}$ pyruvate, BSA $1 \mathrm{mg} / \mathrm{ml}, \mathrm{pH} 7.4$ ) to concentration $5 \times 10^{7} \mathrm{sperm} / \mathrm{ml}$. Experimental sperm samples were treated by estrogens to final concentrations $1 \mathrm{nM}-100 \mu \mathrm{M}$ and control samples with the same amount of ethanol as in the experimental samples. Sperm suspension was incubated for the relevant time $(30,60$, $90,120,180,240 \mathrm{~min}$ ) under paraffin oil at $37^{\circ} \mathrm{C}, 5 \% \mathrm{CO}_{2}$. After $240 \mathrm{~min}$ of incubation, selected samples were treated by boar solubilized zona pellucida (ZP) (Czech Univerzity of Life Sciences, Prague, Czech Republic) for $30 \min \left(37^{\circ} \mathrm{C}, 5 \% \mathrm{CO}_{2}\right)$.

\section{CTC assay}

The chlortetracycline (CTC) fluorescence assay was described previously $[20,21]$. After the capacitation process, sperm suspensions were centrifuged: the capacitation medium was removed and refrigerated for biochemical assays. Sperm were re-suspended in phosphate-buffered saline (PBS) and mixed with equal volume $(45 \mu \mathrm{l} / 45 \mu \mathrm{l})$ of CTC solution $(750 \mathrm{mmol} / \mathrm{l}$ CTC in 130 $\mathrm{mmol} / \mathrm{l} \mathrm{NaCl}, 5 \mathrm{mmol} / \mathrm{l}$ cysteine, $20 \mathrm{mmol} / \mathrm{l}$ Tris- $\mathrm{HCl}, \mathrm{pH}$ 7.8) and incubated for $30 \mathrm{~min}$. Cells were then fixed by 8 $\mu \mathrm{l}$ of $12.5 \%$ paraformaldehyde in $0.5 \mathrm{~mol} / \mathrm{l}$ Tris- $\mathrm{HCl}(\mathrm{pH}$ 7.4). After incubation, sperm suspension was placed on a glass slide, smeared and overlaid by a cover slip. To avoid evaporation and CTC fading, slides were kept in a wet chamber until the evaluation was carried out. Samples were examined with a Nikon Labothot-2 fluorescent microscope equipped with a 40× Nikon Plan 40/0.65 and photographed with a COHU 4910 CCD camera (Inc. 
Electronics Division, San Diego, USA) with the LUCIA imaging software (Laboratory Imaging Ltd., Prague, Czech Republic). Sperm were classified according to their acrosomal staining patterns: (A) Bright fluorescence over the entire sperm head and positive mid-piece of the tail uncapacitated, acrosome intact sperm; (B) Prominent fluorescent positive equatorial segment, mid-piece of the tail and fluorescence-free (dark) band in the postacrosomal region - capacitated, acrosome-intact sperm; (C) Low fluorescent signal throughout the sperm head, with remaining positive signal in the equatorial segment and mid-piece - acrosome-reacted sperm (Fig. 1). Sperm with a nonspecific or intermediate fluorescent signal status were not selected for subsequent analysis. In each sample, 200 cells were evaluated and the minimal number of evaluated samples was 5 .

\section{Indirect immunofluorescence with anti-acrosin ACR.2 monoclonal antibody}

ACR.2 immunofluorescent analysis was described previously $[22,23]$. After the capacitation process, sperm suspensions were centrifuged; the capacitation medium was removed, and kept at $-20^{\circ} \mathrm{C}$. Sperm were re-suspended in equal volume of phosphate-buffered saline (PBS), smeared onto glass slides, dried and kept at $4^{\circ} \mathrm{C}$. During fluorescent specimen preparation, sperm slides were fixed with acetone for $10 \mathrm{~min}$, rinsed with PBS, treated with ACR.2 monoclonal antibody and incubated in a wet chamber for $60 \mathrm{~min}$ at $37^{\circ} \mathrm{C}$. After thorough washing in PBS, the smears were treated with FITC-conjugated antimouse IgG antibody (Sigma, Prague, Czech Republic) and again incubated in a wet chamber for $60 \mathrm{~min}$ at $37^{\circ} \mathrm{C}$. After washing in PBS and water, smears were mounted by the Vectashield mounting medium with DAPI (Vector Lab., Burlingame, CA). Samples were examined with a Nikon Labothot-2 fluorescent microscope equipped with 40× Nikon Plan 40/0.65 and photographed with a COHU 4910 CCD camera (Inc. Electronics Division, San Diego,
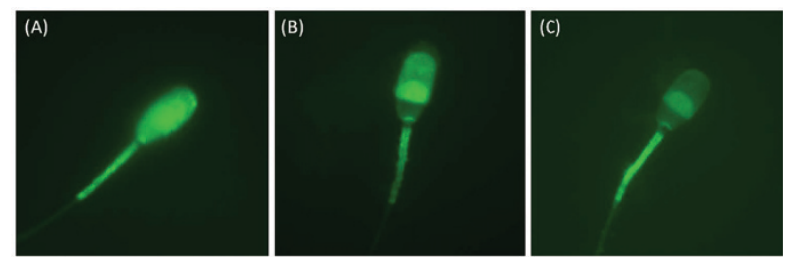

Figure 1 CTC acrosomal fluorescent patterns. Representative pictures of three specific CTC acrosomal fluorescent patterns. (A) Uncapacitated, acrosome intact sperm - bright fluorescence over the entire sperm head and positive mid-piece of the tail; (B) Capacitated, acrosome-intact sperm - prominent fluorescent positive equatorial segment and mid-piece of the tail, fluorescence-free (dark) band in the post-acrosomal region; (C) Acrosome-reacted sperm - low fluorescent signal throughout the sperm head, with a remaining positive signal in the equatorial segment and mid-piece.
USA) using LUCIA imaging software (Laboratory Imaging Ltd., Prague, Czech Republic). Sperm were classified according to their acrosomal staining patterns. (A) Moderate fluorescence in the acrosomal area - uncapacitated, acrosome intact sperm; (B) Intensive fluorescence of the acrosome -- capacitated, acrosome-intact sperm; (C) Low or no fluorescent signal in the sperm head with a remaining positive equatorial segment - acrosome-reacted sperm (Fig. 2). Sperm with nonspecific or intermediate acrosomal status were not selected for subsequent analysis. In each sample, 200 cells were evaluated and the minimal number of evaluated samples was 5 .

\section{Flow cytometry analysis with ACR.2 antibody}

The control, capacitated and experimental sperm samples from animals with high responsiveness to $E$ (animal A) were influenced by $1 \mu \mathrm{M}$ E2, then washed in PBS and fixed by $96 \%$ ethanol at $4^{\circ} \mathrm{C}$ for $60 \mathrm{~min}$. After ethanol fixation, sperm were refixed in ethanol-acetone mixture at $4^{\circ} \mathrm{C}(1: 1)$ for $30 \mathrm{~min}$. Sperm were then washed three times in PBS and incubated with anti-acrosin ACR.2 antibody $(50 \mu \mathrm{g} / \mathrm{ml})$ at $37^{\circ} \mathrm{C}$ for $60 \mathrm{~min}$. After the incubation with the primary antibody, sperm were washed three times in PBS and incubated with a secondary anti-mouse IgG antibody (Sigma, Prague, Czech Republic). After the incubation sperm samples were intensively washed in PBS (five times for $5 \mathrm{~min}$ ) then $100 \mu \mathrm{l}$ of the suspension was placed on 96-well plate. Acquisition and analysis were performed on BD LSR II instrument (BD, Becton Drive Franklin Lakes, NJ, USA), excitation laser $488 \mathrm{~nm}$, emission filters 530/40, measurement of fluorescent intensity in FITC channel. Analysis was performed using FlowJo 7.5.4. software (TreeStar Inc., Ashland, OR, USA). The differences among control and experimental samples in arithmetic mean of the fluorescent intensity in the FITC channel were assessed.

\section{Indirect ELISA with ACR.2 antibody}

After in vitro capacitation, sperm samples were centrifuged and sperm-free capacitating medium was collected
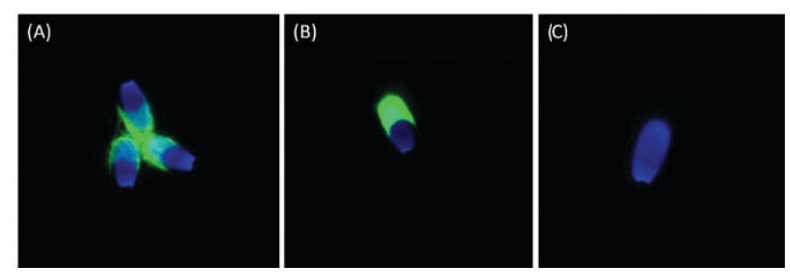

Figure 2 ACR.2 Acrosomal fluorescent patterns (FITC-conjugated secondary antibody). Representative pictures of three specific ACR.2 acrosomal fluorescent patterns. (A) Uncapacitated, acrosome intact sperm - moderate uniform fluorescence in the acrosomal area; (B) Capacitated, acrosome-intact sperm - intensive fluorescence of the acrosome; (C) Acrosome-reacted sperm - low or no fluorescent signal in the sperm head. Nuclei stained with a Blue DAPI dye. 


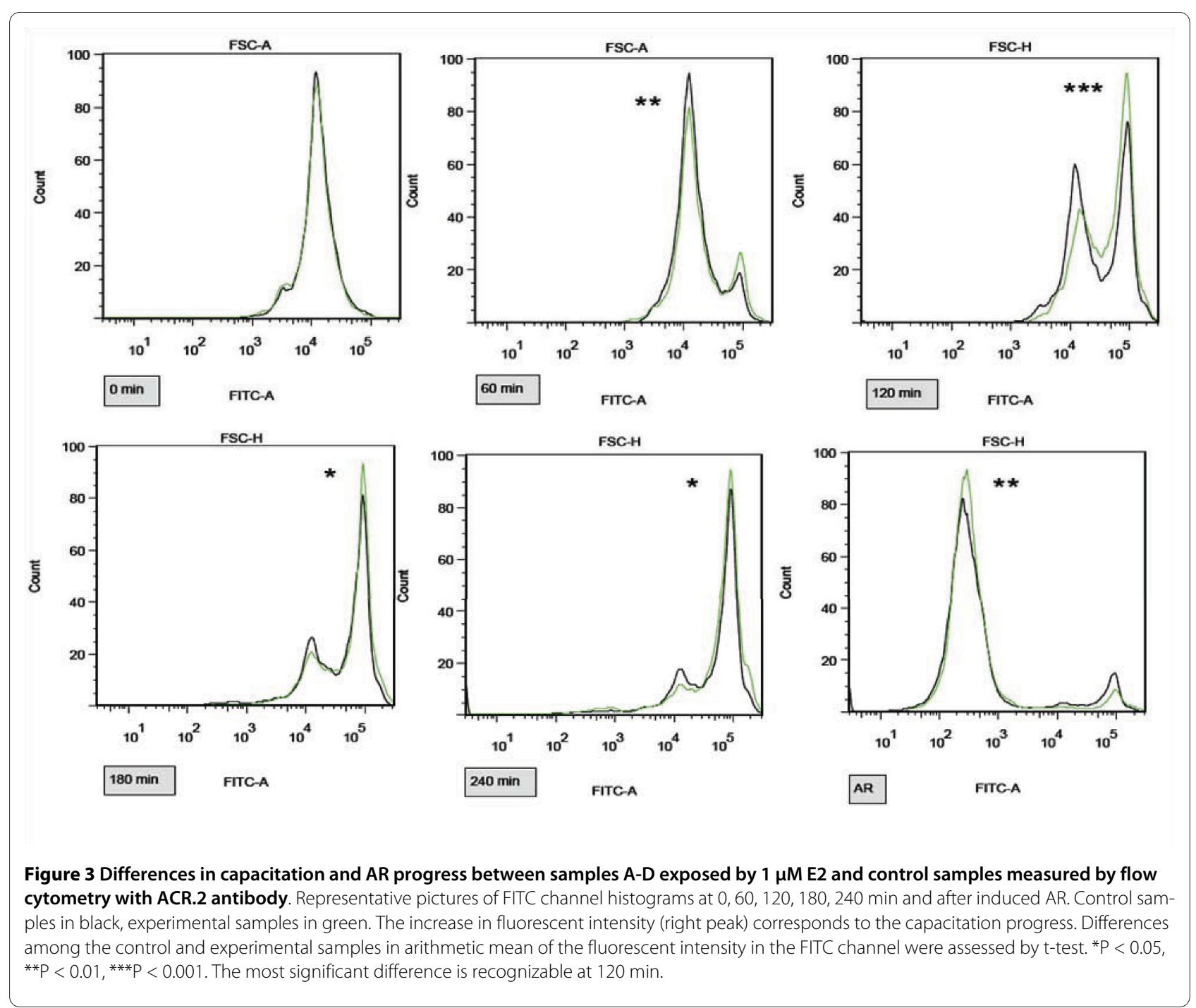

for subsequent biochemical analysis. Capacitation medium was lyophilised and dissolved in determined volume of water. $100 \mu \mathrm{l}$ of the dissolved lyophilisate was applied on a microtiter plate and incubated for 24 hours. After one-day of incubation, the plate was washed three times by PBS and PBS-TWEEN (2\%). The cells were treated by ACR.2 monoclonal antibody [22] and incubated for $60 \mathrm{~min}$. After incubation with primary ACR.2 antibody, the plate was washed and treated with peroxidase-conjugated swine anti-mouse antibody (SWAM-Px, Sevapharma, Prague, Czech Republic) conjugated and incubated for $30 \mathrm{~min}$. After the second incubation, the plate was washed, and cells were treated by $o$-phenylenediamine (Fluka, Buchs, Switzerland) for $3 \mathrm{~min}$. The reaction was stopped by $4 \mathrm{~N}$ sulfuric acid and the absorbance was measured on Biotrak II Plate Reader (Amersham Biociences) at $492 \mathrm{~nm}$.

\section{Acrosin activity assay}

After the in vitro capacitation process, sperm samples were centrifuged and sperm-free capacitating medium was collected for subsequent analysis. Capacitation medium was lyophilised and redissolved in $100 \mu \mathrm{l}$ of reaction buffer (0.2 M Tris. $\mathrm{HCl}, 0.02 \mathrm{M} \mathrm{CaCl} 2, \mathrm{pH}=8)$, placed on a microtiter plate and incubated for $10 \mathrm{~min}$. After the first incubation the BAPA solution (1 mg N $\alpha$ Benzoyl-L-arginine 4-nitroanilide hydrochloride/1 ml dimethylformamide) was added and this was incubated for $20 \mathrm{~min}$. After the second incubation, the reaction was stopped by $30 \%$ formic acid and the absorbance of samples was measured on a Biotrak II Plate Reader (Amersham Biociences) at $405 \mathrm{~nm}$.

\section{Statistical analysis}

Experimental data were analyzed using STATISTICA 7.0. (StatSoft CR, Prague, Czech Republic). The statistical differences in the number of sperm with specific acrosomal status among control and experimental samples were assessed by the Kruskal-Wallis one-way analysis of variance (KW-ANOVA). Statistical differences between the continuous values (arithmetic means of the fluorescent intensity in the FITC channel in flow cytometry analysis, 

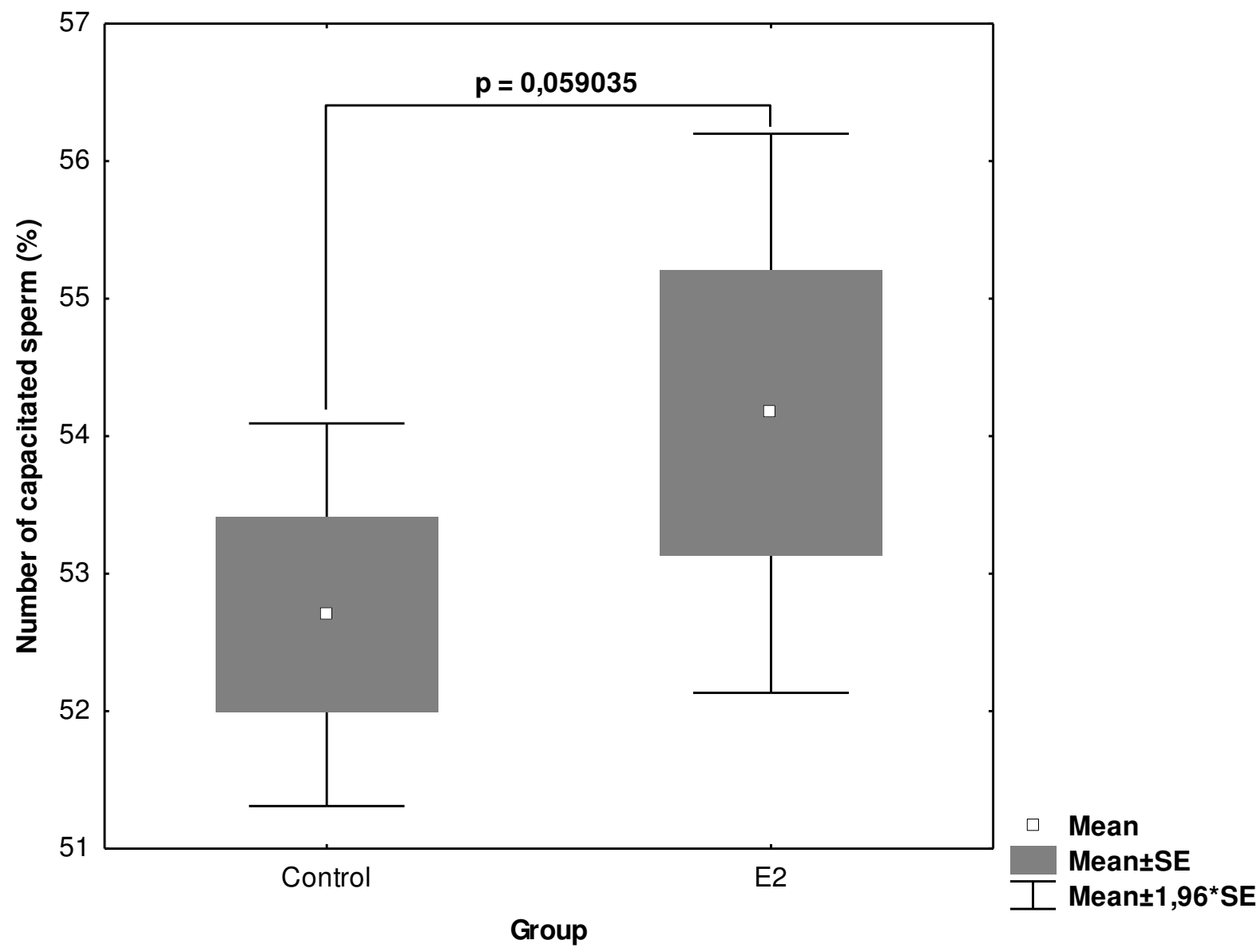

Figure 4 Differences in the number of capacitated sperm in control and experimental samples influenced by $1 \mu \mathrm{M}$ E2 in 8 individual animals. Sperm samples from 8 individual animals were capacitated with $1 \mu \mathrm{M}$ E2 and ethanol (control). Sperm were collected after 120 min of capacitation and analyzed by CTC and ACR.2 immunofluorescence. Differences were analyzed by Mann-Whitney $U$ test ${ }^{*} P<0.05$, ${ }^{* * P}<0.01$, ***P $<0.001$.

absorbance in indirect ELISA with ACR.2 antibody and acrosin activity assay) were assessed by one-way analysis of variance ANOVA. Post hoc analysis was performed by the Newman-Keuls test and multiple comparisons of mean ranks. The $\mathrm{P}$ value, ${ }^{*} \mathrm{P}<0.05,{ }^{* *} \mathrm{P}<0.01$, ${ }^{* * * *} \mathrm{p}<$ 0.001 .

\section{Results}

ACR.2 flow cytometry analysis of $1 \mu \mathrm{M}$ E2 effect on capacitation at selected capacitation times

In order to determine the potential differences in the capacitation progress between control and experimental groups, sperm samples were analyzed by flow cytometry with ACR.2 antibody at selected times of capacitation. The experimental sample was capacitated with E2 at a 1 $\mu \mathrm{M}$ concentration, and the control sample with the same amount of ethanol as in the experimental sample. $1 \mu \mathrm{M}$ concentration of E2 was selected based on the previous mouse study [18] where it was defined as the lowest con- centration with any significant effect on sperm capacitation. Sperm were collected at 0,10,30, 60, 120, 180, and $240 \mathrm{~min}$ of capacitation and after the induced acrosomal reaction. The first significant difference between the control and experimental group was at $60 \mathrm{~min}$ capacitation in the arithmetic mean of the fluorescent intensity in the FITC channel (Fig. 3). The strongest significant difference was then at $120 \mathrm{~min}$ of capacitation. After an induced acrosomal reaction, a significantly higher number of sperm underwent AR in the experimental group in comparison with the control one.

\section{Analysis of $1 \mu \mathrm{M}$ E2 effect on capacitation by CTC fluorescence assay and anti-acrosin ACR.2 monoclonal antibody}

Sperm samples from 8 animals were capacitated in parallel, in the presence of experimental concentration of $1 \mu \mathrm{M}$ E2 and ethanol (control) collected after 120 min of capacitation and analyzed by CTC and ACR.2 immunofluorescence. Only highly correlated results were used in the 


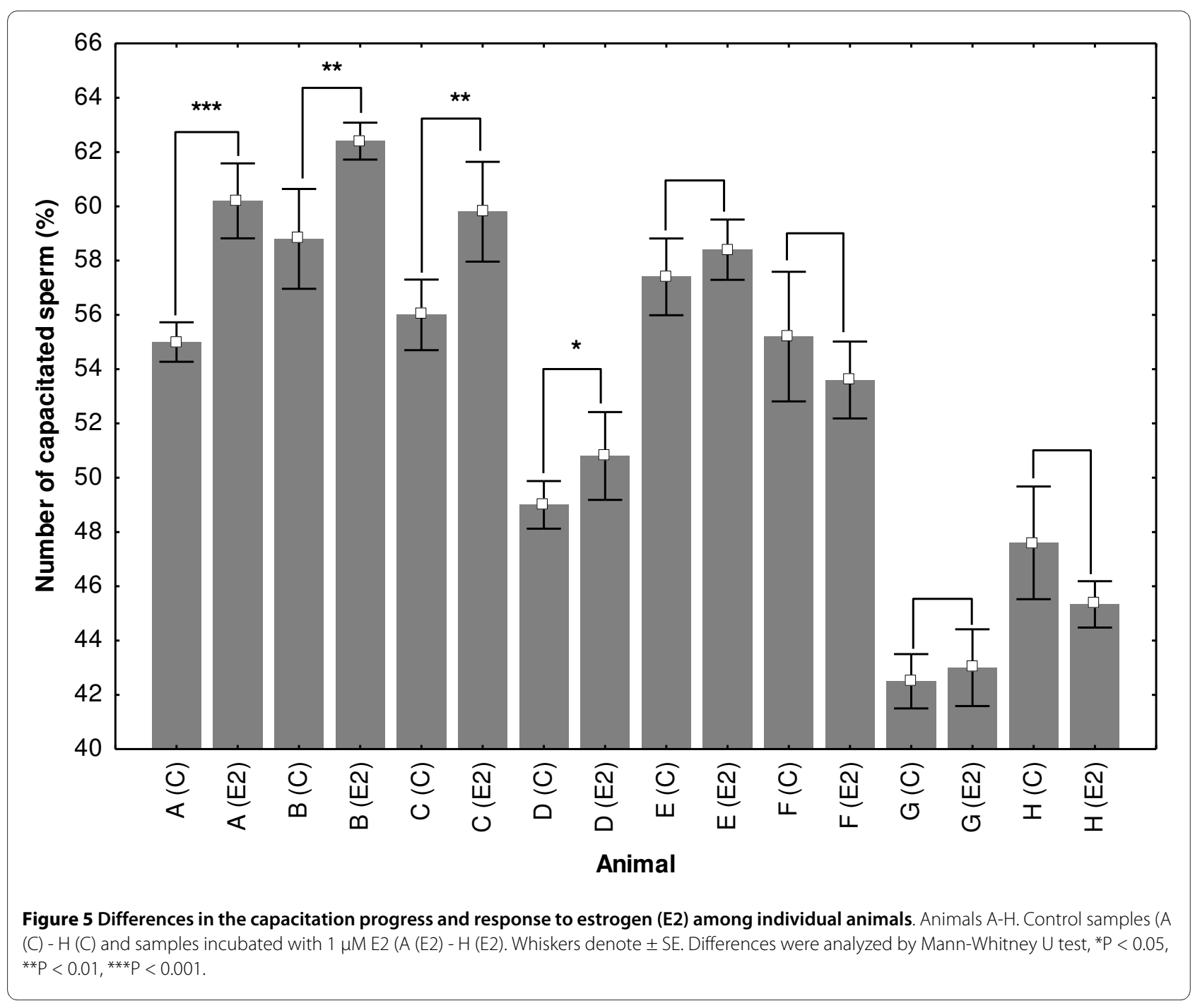

subsequent statistical analysis. E2 has almost significant $(\mathrm{p}=0.059)$ procapacitation effect on boar sperm (Fig. 4). In order to evaluate the potential differences in the capacitation progress and the responsiveness to E2 among individual animals the samples from each boar were analyzed separately. There were significant differences in the capacitation progress and responsiveness to estrogens among individual animals (Fig. 5). In 4 samples, E2 significantly increased the number of capacitated sperm; in 4 other samples, E2 had no significant effect on the capacitation progress.

\section{CTC and ACR.2 analysis of the different estrogen- concentration effect at 120 min capacitation in samples with high response to $\mathrm{E} 2$ (boar $\mathrm{A}$ ) and no significant response to $1 \mu \mathrm{M}$ E2 (boar E)}

Since the most significant difference between the control and E2-influenced experimental group was at $120 \mathrm{~min}$ of capacitation, sperm samples from boar A (with a high responsiveness to E2) and from boar E (with no signifi- cant response to $1 \mu \mathrm{M}$ E2) were capacitated with a different concentration of four estrogens and analyzed by CTC and immunocytochemistry with ACR.2. In each group, at least 5 samples were analyzed. In CTC assay and immunocytochemistry with ACR.2 antibody, only highly correlated results (difference $<5 \%$ ) were used in the subsequent statistical analysis. In the boar A sample, all estrogens significantly accelerate the capacitation progress in a concentration-dependent manner (Tab. 1). E2 showed the first significant effect at $10 \mathrm{nM}$ concentration. All selected estrogens significantly accelerate the capacitation progress at $100 \mathrm{nM}$ concentration. In sperm samples from boar E, only high concentrations of estrogens (10-100 $\mu \mathrm{M})$ stimulated the capacitation progress (Tab. 2).

Analysis of the differences in the number of sperm after ZPinduced AR incubated with $1 \mu \mathrm{M}$ estrogens

Sperm samples were analyzed after 240 min of capacitation and induced AR by CTC, immunocytochemistry and 
Table 1: Number of capacitated sperm in control and experimental samples after 120 of capacitation

\begin{tabular}{|c|c|c|c|c|c|c|c|}
\hline Group & Control & $1 \mathrm{nM}$ & $5 \mathrm{nM}$ & $10 \mathrm{nM}$ & $100 \mathrm{nM}$ & $1 \mu \mathrm{M}$ & $10 \mu \mathrm{M}$ \\
\hline E1 & $55.00 \pm 1.56$ & $55.5 \pm 0.93$ & $55.45 \pm 1.79$ & $56.25 \pm 1.28$ & $57.60 \pm 1.26 * *$ & $59.75 \pm 1.58 * * *$ & $61.50 \pm 0.93 * * *$ \\
\hline E2 & $55.00 \pm 1.56$ & $56.00 \pm 1.41$ & $56.74 \pm 1.21 *$ & $56.80 \pm 1.62^{*}$ & $57.73 \pm 1.27 * * *$ & $60.20 \pm 1.93 * * *$ & $62.00 \pm 1.41 * * *$ \\
\hline E3 & $55.00 \pm 1.56$ & $54.88 \pm 1.36$ & $55.14 \pm 1.75$ & $56.14 \pm 1.35$ & $57.00 \pm 0.89 * *$ & $59.78 \pm 1.48 * * *$ & $61.71 \pm 0.76 * * *$ \\
\hline EE2 & $55.00 \pm 1.56$ & $55.44 \pm 1.81$ & $54.99 \pm 2.25$ & $55.80 \pm 1.93$ & $56.82 \pm 2.04 *$ & $58.30 \pm 2.91 * *$ & $62.00 \pm 1.41 * * *$ \\
\hline
\end{tabular}

Sperm samples from boar A (with high responsiveness to E2) were capacitated in the presence of six different concentrations (1 nM - $10 \mu \mathrm{M})$ of four estrogens and analyzed by CTC and immunocytochemistry with ACR.2. In each group, at least 5 samples were analyzed. In CTC assay and immunocytochemistry with ACR.2 antibody, only highly correlated results (difference $<5 \%$ ) were used in the subsequent statistical analysis. All estrogens significantly accelerated the capacitation progress in a concentration-dependent manner. E2 had the first significant effect at $10 \mathrm{nM}$ concentration, other three estrogens at $100 \mathrm{nM}$ concentration. Differences were analyzed by KW-ANOVA; post hoc comparison was performed by multiple comparisons of mean ranks. ${ }^{*} \mathrm{P}<0.05,{ }^{* *} \mathrm{P}<0.01,{ }^{* * * P}<0.001$.

ELISA with ACR.2 antibody. The acrosin assay was used to further evaluate the effect of estrogens on capacitation and the acrosomal reaction. There was a significantly higher number of sperm, which underwent ZP -induced $A R$ in all experimental samples in comparison with the experimental group (Fig. 6). Data from immunocytochemistry were further verified by ELISA with ACR.2 antibody and the acrosin assay (Fig. 7).

\section{Discussion}

In this study, we addressed several questions concerning the effect of estrogens on boar sperm in vitro. Although several previous studies have reported on the effects of estrogen in mature spermatozoa among different species, there are some contradictory results in this field. Therefore we employed multiple evaluation techniques to complexly analyze the effect of estrogen on boar sperm in vitro. The obtained results from each method might be useful whilst searching for specific mechanisms, which mediate the estrogen effect in mammalian sperm.

The first experiment addressed the question of whether strong, naturally occurring estrogen (E2) has a significant impact on the boar sperm capacitation progress at differ- ent capacitation times. Sperm were capacitated in vitro in the presence of $1 \mu \mathrm{M}$ E2 or ethanol (control). We found out that $1 \mu \mathrm{M}$ E2 has a procapacitation effect on the boar sperm in vitro. Furthermore, we demonstrated the nonidentical effect of E2 on capacitation at different times of incubation. The first significant difference between the control and experimental samples was at $60 \mathrm{~min}$, and the strongest response was at $120 \mathrm{~min}$ of capacitation. In the later capacitation stages 180 min onwards, the difference between the control and experimental samples was not significant. The observed time-dependent effect of estrogens on the capacitation process is an important finding. In previous publications, authors analysed sperm capacitation status after $30 \mathrm{~min}$ [18], 180 - $300 \mathrm{~min}$ [16] and 360 min [14] and this fact might be an important source for some of the contradictory results. Therefore, the effect of estrogens on capacitation of mammalian sperm should be analyzed at carefully selected capacitation times reflecting the status of the ongoing sperm capacitation process in individual species. Furthermore, the analysis of the time-dependent effect of estrogens on capacitation might be useful while searching for specific molecular processes, which are temporally correlated with the most sig-

Table 2: Number of capacitated sperm in control and experimental samples after 120 of capacitation

\begin{tabular}{|c|c|c|c|c|c|c|c|}
\hline Group & Control & $1 \mathrm{nM}$ & $10 \mathrm{nM}$ & $100 \mathrm{nM}$ & $1 \mu \mathrm{M}$ & $10 \mu \mathrm{M}$ & $100 \mu \mathrm{M}$ \\
\hline E1 & $57.4 \pm 1.14$ & $57.5 \pm 1.89$ & $57.55 \pm 1.78$ & $57.37 \pm 2.62$ & $58.11 \pm 1.58$ & $60.12 \pm 1.12 * *$ & $61.25 \pm 1.56 * * *$ \\
\hline E2 & $57.4 \pm 1.14$ & $58.00 \pm 1.61$ & $58.80 \pm 1.62$ & $58.11 \pm 2.11$ & $58.40 \pm 0.89$ & $60.10 \pm 1.22 * *$ & $61.81 \pm 1.22 * * *$ \\
\hline E3 & $57.4 \pm 1.14$ & $56.89 \pm 1.75$ & $56.14 \pm 1.35$ & $57.00 \pm 1.76$ & $57.78 \pm 2.45$ & $60.22 \pm 1.56^{* *}$ & $61.54 \pm 1.67^{* * *}$ \\
\hline EE2 & $57.4 \pm 1.14$ & $57.84 \pm 2.09$ & $57.70 \pm 1.93$ & $58.42 \pm 1.36$ & $58.30 \pm 1.98$ & $60.10 \pm 2.31^{* *}$ & $61.43 \pm 1.37 * * *$ \\
\hline
\end{tabular}

Sperm samples from boar $\mathrm{E}$ (with no significant responsiveness to $\mathrm{E} 2$ at concentration of $1 \mu \mathrm{M}$ ) were capacitated in the presence of six different concentrations ( $1 \mathrm{nM}-100 \mu \mathrm{M})$ of four estrogens and analyzed by CTC and immunocytochemistry with ACR.2. In each group, at least 5 samples were analyzed. In CTC assay and immunocytochemistry with ACR.2 antibody, only highly correlated results (difference $<5 \%$ ) were used in the subsequent statistical analysis. All estrogens significantly accelerated the capacitation progress at $10 \mu \mathrm{M}$ concentration. Differences were analyzed by KW-ANOVA; post hoc comparison was performed by multiple comparisons of mean ranks. ${ }^{*} \mathrm{P}<0.05,{ }^{* *} \mathrm{P}<0.01,{ }^{* * *} \mathrm{P}<0.001$. 


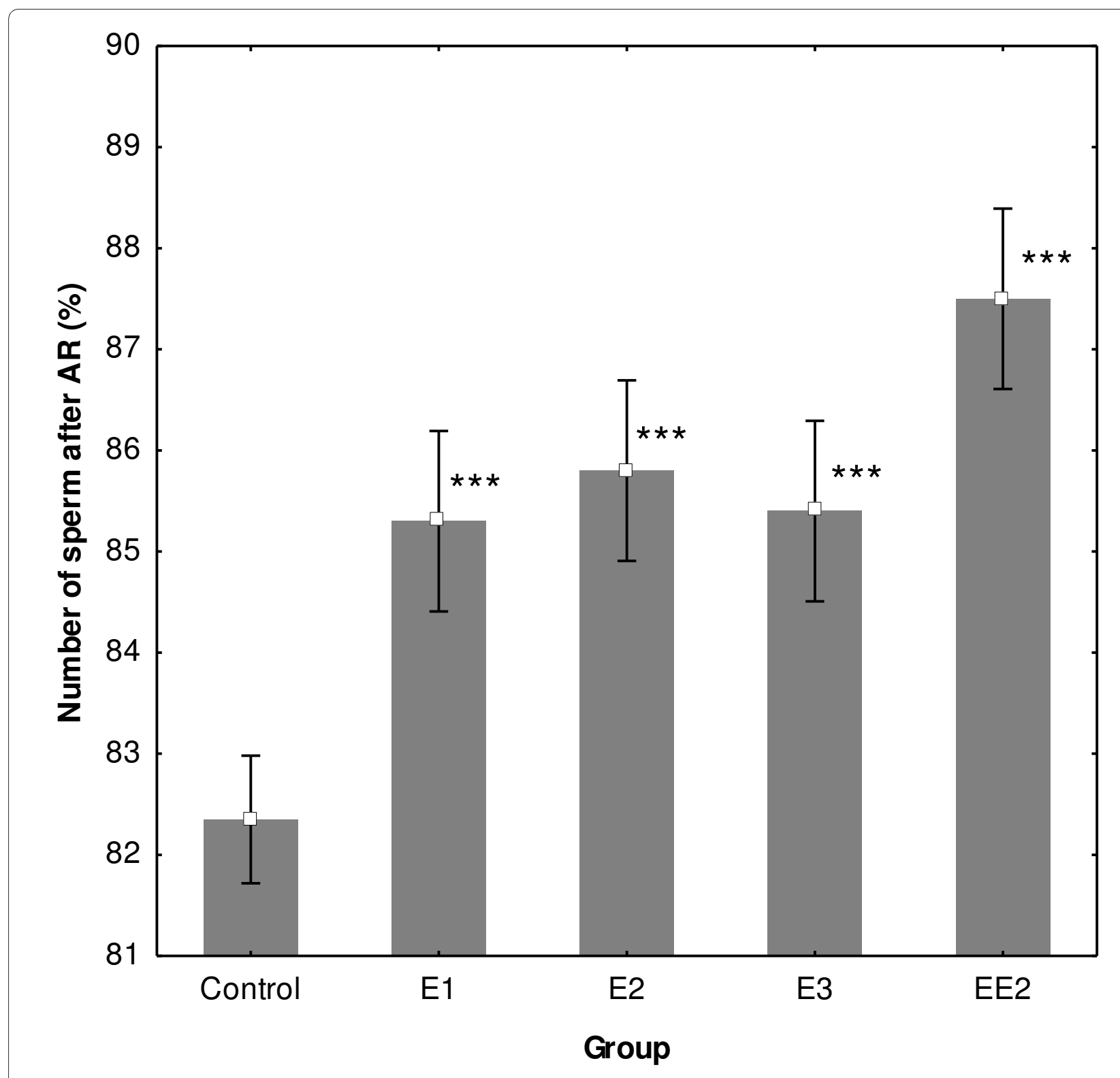

Figure 6 Number of sperm after AR evaluated by ACR.2 antibody. After 240 min of capacitation, control and experimental samples from boars A-D with $1 \mu \mathrm{M}$ concentration of four estrogens were treated by zona pellucida to induce AR. There was a significantly higher number of sperm, which underwent calcium ZP-induced AR in all experimental samples in comparison to the experimental group. All estrogens significantly increased the number of sperm after the ZP-AR. Differences were analyzed by KW-ANOVA; post hoc comparison was performed by multiple comparisons of mean ranks. Whiskers denote $\pm \mathrm{SE}$. ${ }^{*} \mathrm{P}<0.05,{ }^{* * P}<0.01,{ }^{* * * P}<0.001$.

nificant effect of estrogens (e.g. calcium influx, cholesterol efflux, actin polymerisation, protein phosphorylation, acrosomal rearrangement etc.) [24].

In the second experiment, we wondered whether E2 has a similar effect on sperm samples collected from different individuals. We observed strong differences in the response to estrogens among samples from different individual animals during capacitation in vitro. According to our results, the analysis of different responsiveness to estrogens among individual animals in the tested popula- tion might be important, because individual variability strongly affects general results. Furthermore, a detailed analysis of the individuals with high and low estrogenresponsiveness can elucidate the mechanism of the estrogen action in sperm. Hitherto, there is no plausible parameter e.g. expression of a different estrogen receptor correlating with estrogen responsiveness [25].

In the third experiment, we tested the effect of four different estrogens on the capacitation progress of sperm collected from boar with high and no significant differ- 


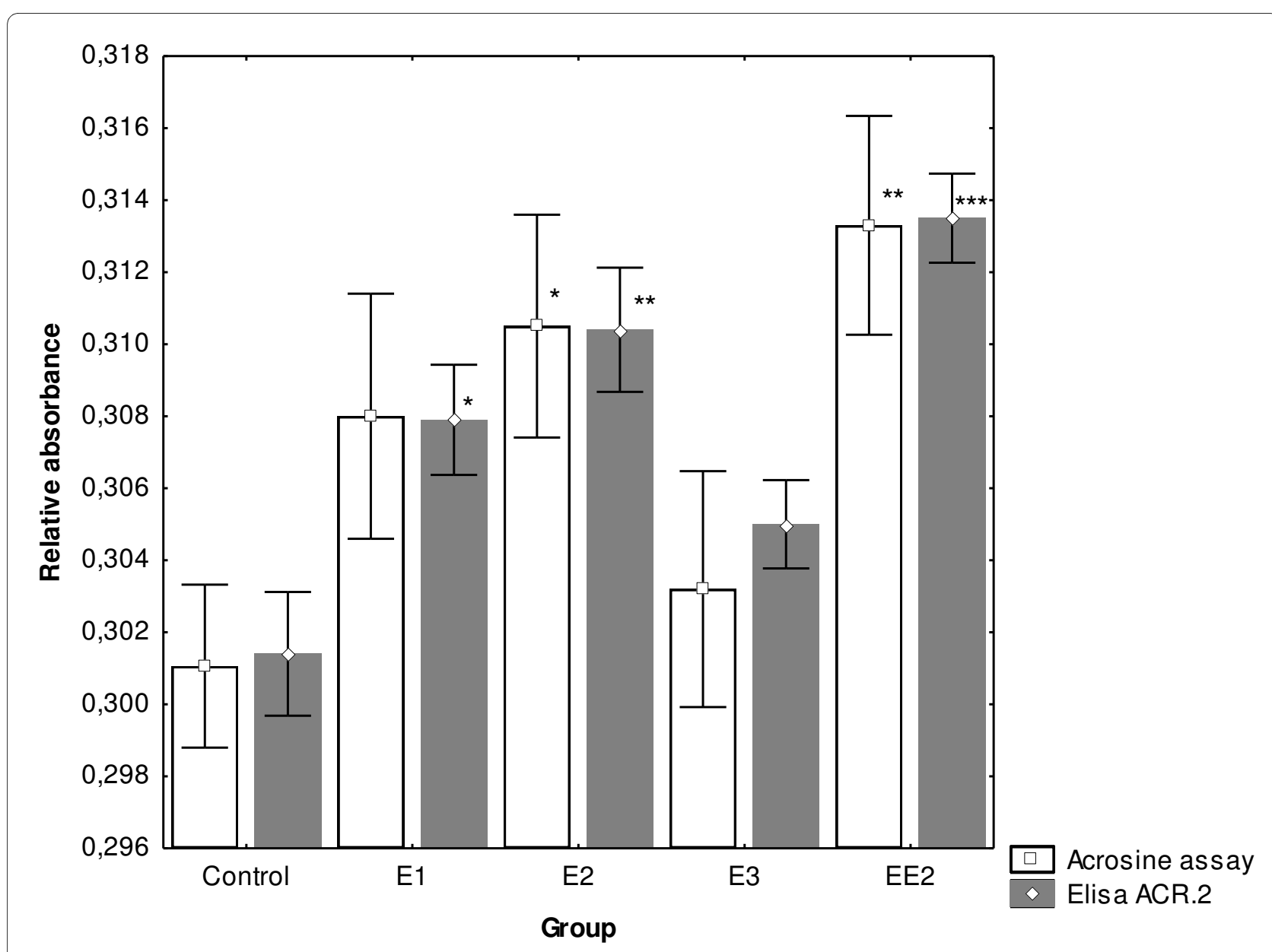

Figure 7 Concentration of acrosin in medium after induced AR measured enzymatically by acrosin assay and immunochemically by ELISA with ACR.2 antibody. After 240 min of the in vitro capacitation process, experimental samples from boars A-D with $1 \mu$ M concentration of four estrogens were treated by boar zona pellucida to induce AR. Capacitation media were analyzed by ELISA with ACR.2 antibody and acrosin assay to biochemically determine the number of sperm after AR. Differences were analyzed by ANOVA; post hoc comparison was performed by Newman-Keuls test. Whiskers denote $\pm \mathrm{SE}$. ${ }^{*} \mathrm{P}<0.05,{ }^{* * \mathrm{P}}<0.01,{ }^{* * *} \mathrm{P}<0.001$.

ence. Analysis of the effect of multiple compounds with a similar physiological effect (E1, E2, E3, EE2) provides more reliable data than analysis based only on one compound. In an animal with high estrogen-responsiveness, estrogens stimulate capacitation in a concentration dependent manner. E2 has a significant effect at $5 \mathrm{nM}$ concentration; all other estrogens have a significant effect at $100 \mathrm{nM}$ concentration. Although contrary to other estrogens, E2 had a significant effect at $5 \mathrm{nM}$ and $10 \mathrm{nM}$ concentrations. There was no significant difference between individual estrogens at the appropriate concentration level. In the boar sample with no significant response to $1 \mu \mathrm{M}$ E2, only a very high concentration of estrogens stimulates capacitation $(10-100 \mu \mathrm{M})$. It suggests that estrogens have a general procapacitation effect, but in some animals, the responsiveness to estrogens is low and only very high concentrations of estrogens are able to provoke a procapacitation effect. The differences in estrogen-responsiveness further suggest that multiple mechanisms in the estrogen action in sperm might be involved. In the samples with high responsiveness, estrogens (E2) have a significant effect at concentrations normally required for estrogen receptor-mediated cellular response $[26,27]$. The fact that a higher concentration of estrogens at which estrogen receptors are almost saturated will still increase the number of capacitated cells in a concentration-dependent manner suggests that the estrogen effect at high concentration might be mediated by another, nonreceptor mechanism (membrane changes, etc.) [25]. This idea is further supported by the fact that in samples with no significant response to $1 \mu \mathrm{M}$ E2, estrogens have a significant effect at high concentrations (10$100 \mu \mathrm{M}$ ), which are far from a concentration needed for the estrogen receptor mediated cellular response in somatic cells. This fact suggests that the specific mechanism (e.g. receptor signalization), which is responsible for 
estrogen responsiveness at low concentration, is not functional in samples with no response to 1-10 nM E2. Nevertheless, high experimental concentrations of estrogens $(10-100 \mu \mathrm{M})$ are far from the physiological plasma levels of estrogens (e.g. $10^{-10}-10^{-11} \mathrm{M}$ for E2 in rats and mice [28]). However, the concentration of estrogens in follicular fluid is on the other hand higher [28] and sperm may be, therefore, exposed to high concentrations during their capacitation in the female reproductive tract [29,30].

Finally, in the last experiment, we demonstrated the significant impact of estrogens on the ZP-induced acrosomal reaction. The number of sperm after AR was significantly higher in all experimental groups. The induced acrosomal reaction data were evaluated microscopically and also confirmed by objective biochemical methods. Therefore, the results obtained from ZPinduced acrosomal reaction, not only confirm the capacitation experiment, but also suggest that estrogens have a real physiological impact on sperm capacitation, as the analysis was based, in particular, on molecular and cellular markers of capacitation (calcium influx, acrosomal rearrangement). Furthermore, the analysis by objective biochemical methods (ELISA, acrosin assay) provides an important supporting data to the subjective microscopical evaluation methods.

In conclusion, in this study we addressed several important questions concerning the effect of estrogens on boar sperm capacitation in vitro. We found out that in boar sperm in vitro estrogens generally show a procapacitation effect. This effect depends strongly on the stage of the capacitation progress, estrogen concentration and individual responsiveness of tested animals. Individual estrogens have a relatively similar effect. These observations have a significant impact on our understanding of the previous results concerning estrogen effects in sperm and should be helpful to uncover the specific mechanisms of the estrogen effects in sperm physiology.

\section{Competing interests}

The authors declare that they have no competing interests.

\section{Authors' contributions}

LD is responsible for sperm capacitation, analysis of the capacitation process by all described methods, for statistical analysis and preparation of the manuscript. PD is responsible for the preparation of the media with specific concentrations of estrogens, sperm capacitation, analysis of sperm capacitation status by CTC and immunocytochemistry by ACR.2 antibody. AD is responsible for sperm capacitation and analysis of capacitation media after the induced AR. JP and $\mathrm{KH}$ are responsible for the conception of the study, coordination of experiments, revising and final approval of the manuscript. All authors read and approved the final manuscript.

\section{Acknowledgements}

The work was supported by Grants of the Ministry of Education of the Czech Republic Nos. VC 1 M06011 and VZ 0021620828, the Grant Agency of the Czech Republic Nos. 523/08/H064 and 523/09/1793, and by the Institutional Research Support AVOZ 50520701. We are thankful to Timothy Paul Hort for English corrections.

\section{Author Details}

${ }^{1}$ Laboratory of Diagnostics for Reproductive Medicine, Institute of Biotechnology, Academy of Sciences of the Czech Republic, v. v. i., Prague, Czech Republic and 2Department of Zoology, Faculty of Science, Charles University, Prague, Czech Republic

Received: 9 February 2010 Accepted: 13 July 2010

Published: 13 July 2010

\section{References}

1. Austin CR: Observation on penetration of sperm into the mammalian egg. Aust J Sci Res 1951, 4:581-596.

2. Austin CR: Capacitation of spermatozoa. Int J Fertil 1967, 12:25-31.

3. Chang MC: Ferilizing capacity of spermatozoa deposited into the Fallopian tubes. Nature 1951, 168:997-998.

4. Hunter RH, Hall JP: Capacitation of boar spermatozoa: synergism between uterine and tubal environments. J Exp Zool 1974, 188(2):203-213

5. Brown SM, Hamner CE: Capacitation of sperm in the female reproductive tract of the rabbit during estrus and pseudopregnancy. Fertil Steril 1971, 22(2):92-97.

6. Yanagimachi R: In vitro capacitation of hamster spermatozoa by follicular fluid. J Reprod Fertil 1969, 18(2):275-286.

7. Niwa $\mathrm{K}$, Imai H, Kim Cl, Iritani A: Fertilization in vitro of hamster and mouse eggs in a chemically defined medium. J Reprod Fertil 1980, 58(1):109-114.

8. Eddy EM, Washburn TF, Bunch DO, Goulding EH, Gladen BC, Lubahn DB, Korach KS: Targeted disruption of the estrogen receptor gene in male mice causes alteration of spermatogenesis and infertility. Endocrinology 1996, 137(11):4796-4805.

9. Acconcia F, Kumar R: Signaling regulation of genomic and nongenomic functions of estrogen receptors. Cancer Lett 2006, 8; 238(1):1-14

10. Aquila S, Sisci D, Gentile M, Middea E, Catalano S, Carpino A, Rago V, Andò $\mathrm{S}$ : Estrogen receptor (ER) alpha and ER beta are both expressed in human ejaculated spermatozoa: evidence of their direct interaction with phosphatidylinositol-3-OH kinase/Akt pathway. J Clin Endocrinol Metab 2004, 89(3):1443-1451.

11. Naz RK, Sellamuthu R: Receptors in spermatozoa: are they real? J Androl 2006, 27(5):627-636

12. Rago V, Aquila S, Panza R, Carpino A: Cytochrome P450arom, androgen and estrogen receptors in pig sperm. Reprod Biol Endocrinol 2007, 5:23.

13. Luconi M, Francavilla F, Porazzi I, Macerola B, Forti G, Baldi E: Human spermatozoa as a model for studying membrane receptors mediating rapid nongenomic effects of progesterone and estrogens. Steroids 2004, 69(8-9):553-559.

14. Gwatkin RB, Williams DT: Inhibition of Sperm Capacitation in vitro by Contraceptive Steroids. Nature 1970, 227(5254):182-183.

15. Briggs $\mathrm{MH}$ : Steroid hormones and the fertilizing capacity of spermatozoa. Steroids 1973, 22(4):547-553.

16. Bathla H, Guraya SS, Sangha GK: Role of estradiol in the capacitation and acrosome reaction of hamster epididymal spermatozoa in the isolated uterus of mice incubated in vitro. Indian J Physiol Pharmacol 1999, 43(2):211-217.

17. Hamner CE, Wilson LA Jr: Inhibition of capacitation in the rabbit. Fertil Steril 1972, 23(3):196-200.

18. Adeoya-Osiguwa SA, Markoulaki S, Pocock V, Milligan SR, Fraser LR: 17beta-Estradiol and environmental estrogens significantly affect mammalian sperm function. Hum Reprod 2003, 18(1):100-107.

19. Francavilla F, Romano R, Pandolfi C, Macerola B, Santucci R, Necozione S, Francavilla S: Evaluation of the effect of 17alphaOH-progesterone and 17beta-oestradiol on human sperm ability to fuse with oocytes: comparison and possible interference with the effect of progesterone. Int J Andro/ 2003, 26(6):342-347.

20. Wang $W H$, Abeydeera LR, Fraser LR, Niwa K: Functional analysis using chlortetracycline fluorescence and in vitro fertilization of frozenthawed ejaculated boar spermatozoa incubated in a protein-free chemically defined medium. J Reprod Fertil 1995, 104(2):305-313.

21. Fraser $L R$, Abeydeera $L R$, Niwa $K: \mathrm{Ca}(2+)$-regulating mechanisms that modulate bull sperm capacitation and acrosomal exocytosis as determined by chlortetracycline analysis. Mol Reprod Dev 1995, 40(2):233-241. 
22. Peknicová J, Moos J: Monoclonal antibodies against boar acrosomal antigens labelling undamaged acrosomes of spermatozoa in immunofluorescence test. Andrologia 1990, 22(5):427-435.

23. Peknicova J, Moos J, Mollova M, Srsen V, Capkova J: Changes in immunochemical localisation of acrosomal and sperm proteins in boar spermatozoa during capacitation and induced acrosome reaction. Anim Repr Sci 1994, 35:255-271.

24. De Jonge C: Biological basis for human capacitation. Hum Reprod Update 2005, 11(3):205-214

25. Baldi E, Luconi M, Muratori M, Marchiani S, Tamburrino L, Forti G Nongenomic activation of spermatozoa by steroid hormones: facts and fictions. Mol Cell Endocrinol 2009, 308(1-2):39-46.

26. Nawata $\mathrm{H}$, Chong MT, Bronzert D, Lippman ME: Estradiol-independent growth of a subline of MCF-7 human breast cancer cells in culture. $J$ Biol Chem 1981, 256(13):6895-902.

27. Blair RM, Fang H, Branham WS, Hass BS, Dial SL, Moland CL, Tong W, Shi L, Perkins $R$, Sheehan DM: The estrogen receptor relative binding affinities of 188 natural and xenochemicals: structural diversity of ligands. Toxicol Sci 2000:138-153.

28. Nishimura I, Ui-Tei K, Saigo K, Ishii H, Sakuma Y, Kato M: 17-Estradiol at Physiological Concentrations Augments $\mathrm{Ca}^{2+}$-Activated $\mathrm{K}+$ Currents via Estrogen Receptor in the gonadotropin-Releasing Hormone Neuronal Cell Line GT1-7. Endocrinology 2008, 149(2):774-782.

29. Tarlatzis BC, Pazaitou K, Bili H, Bontis J, Papadimas J, Lagos S, Spanos E, Mantalenakis S: Growth hormone, oestradiol, progesterone and testosterone concentrations in follicular fluid after ovarian stimulation with various regimes for assisted reproduction. Hum Reprod 1993, 8(10):1612-1616.

30. Ouellette Y, Price CA, Carrière PD: Follicular fluid concentration of transforming growth factor-beta 1 is negatively correlated with estradiol and follicle size at the early stage of development of the firstwave cohort of bovine ovarian follicles. Domest Anim Endocrinol 2005, 29(4):623-633.

doi: $10.1186 / 1477-7827-8-87$

Cite this article as: Ded et al., Effect of estrogens on boar sperm capacitation in vitro Reproductive Biology and Endocrinology 2010, 8:87

Submit your next manuscript to BioMed Central and take full advantage of:

- Convenient online submission

- Thorough peer review

- No space constraints or color figure charges

- Immediate publication on acceptance

- Inclusion in PubMed, CAS, Scopus and Google Scholar

- Research which is freely available for redistribution

Submit your manuscript at www.biomedcentral.com/submit
Ciomed Central 